\title{
Electro-Mechanical Behavior of the ATLAS Toroids in Fault Condition
}

\author{
Arnaud Foussat, Alexander Abramov, Paolo Luzzatto Fegiz, Alexey Dudarev, Giovanni Volpini, and \\ Herman H. J. ten Kate
}

\begin{abstract}
The three toroids magnet in the ATLAS Detector provides the magnetic field for the muon detection system. It consists of a Barrel Toroid and two End Cap Toroids, which are electrically and magnetically coupled. In order to guarantee a safe operation, the behavior of the toroid support structures have to be verified in fault conditions. The mechanical behavior of the ATLAS magnet system in the equilibrium operating condition has been calculated in the past. In this paper, results of 3D electro-magnetic calculations of unbalanced force distributions under fault conditions are presented and analyzed. In addition, the energy dissipation and acceptable operating ramp rate in the windings are discussed.
\end{abstract}

Index Terms-Magnetic field, mechanical behavior, quench protection, short to ground and neighboring turns, superconducting toroid.

\section{INTRODUCTION}

$\mathbf{T}$ HE ATLAS Detector Magnet System which is being installed in the LHC accelerator at CERN, is composed of 8 racetrack shape barrel toroid coils (BT), 8 end cap toroid coils (ECT) on each side of BT and a Central Solenoid (CS). The overall dimensions of the system are $22 \mathrm{~m}$ outer diameter and $25 \mathrm{~m}$ total length [1]. The nominal magneto-motive force of Barrel Toroid is 19.7 MAt with a peak field in the conductor of 4.4 T. The central solenoid has independent electrical circuit and operates at 8.9 MAt with conductor peak field at 2.3 T. This paper first focuses on the thermal and electromagnetic operating limitations of the ATLAS Barrel Toroid system in the presence of a short circuit. Then, the structural behavior is investigated using the Opera code [2] in order to assess the possibilities for continued operation in an off-design regime. The aim is to study force distributions in the conductors under different fault scenarios.

\section{Circuit Analysis of Fault}

\section{A. Toroids Electrical Circuit}

Previous fault scenario studies have identified main causes of faults [3], like short circuit conditions, and define parameters of circuit discharge in the case of a quench due to shorts. Short circuits can occur between bus bars, between ground and bus bars, inside one or between coils. The presence of shorts in su-

Manuscript received October 21, 2003.

A. Foussat, A. Abramov, P. L. Fegiz, A. Dudarev and H. H. J. ten Kate are with the ATLAS Magnet Team, CERN, Geneva, 1211 Geneva 23 Switzerland (e-mail: arnaud.foussat@cern.ch).

G. Volpini is with INFN sez. Di Milano, Laboratorio LASA, 20090 Segrate, Italy.

Digital Object Identifier 10.1109/TASC.2004.829713
TABLE I

BARREL TOROID COIL MAIN FEATURES

\begin{tabular}{llll}
\hline \hline & Units & BT & one ECT \\
Dimensions & $\mathrm{m}$ & $5 \times 25$ & $5 \times 5$ \\
Cable Cross Section & $\mathrm{mm}^{2}$ & $57 \times 12$ & $41 \times 12$ \\
Current & $\mathrm{A}$ & 20500 & 20500 \\
EM Stored Energy & $\mathrm{MJ}$ & 1080 & 216 \\
Operating Temperature & $\mathrm{K}$ & 4.8 & 4.8 \\
Critical Current (T=4.2K, B=5T) & $\mathrm{A}$ & $>58000$ & $>60000$ \\
Maximum magnetic Field & $\mathrm{T}$ & 3.85 & 4.1 \\
RRR (Al, B=0T) & - & $>1000$ & $>1000$ \\
Weight & tons & $8 \times 95$ & 260 \\
Numbers of turns & & $8 \times 120$ & $8 \times 120$ \\
Ramp down resistors & $\mathrm{m} \Omega$ & 0.62 & 0.16 \\
& & & \\
\hline \hline
\end{tabular}

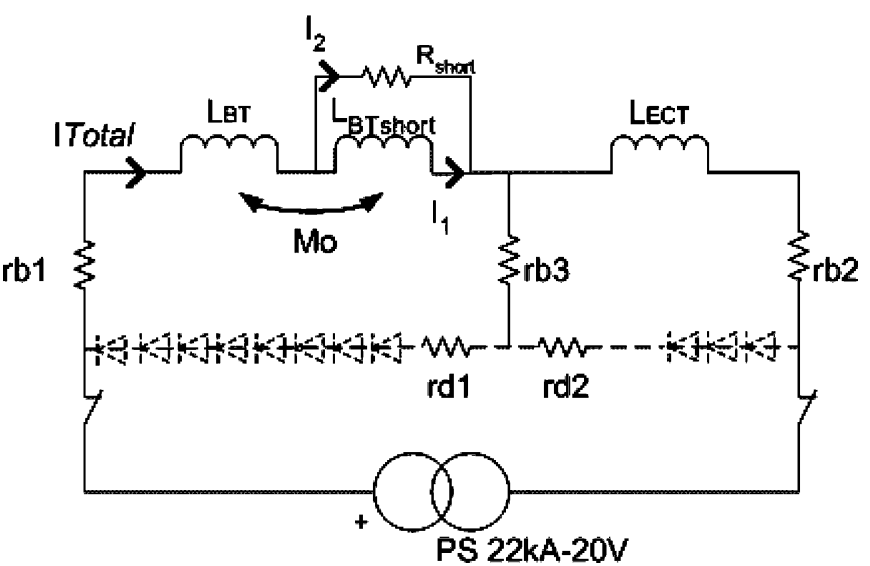

Fig. 1. Electrical circuit of the ATLAS toroids with a short circuit in the barrel toroid.

perconducting coils can result in quench during current ramps. It may limits the ramp rate during charging to unacceptably low values. The main features of the Toroid are listed in Table I. The toroidal coils are supplied by a $20 \mathrm{~V}-22 \mathrm{kA}$ rated power supply, as shown in Fig. 1.

$\mathrm{L}_{1}$ is the inductance of the main nonshorted section, composed of $\mathrm{L}_{\mathrm{BT}}$, sum of the entire $\mathrm{BT}$ inductances and $\mathrm{L}_{\mathrm{ECT}}$ the inductances of $\mathrm{ECT}_{\mathrm{A}}$ and $\mathrm{ECT}_{\mathrm{C}}$. $\mathrm{L}_{2}$ is the shorted section of the Barrel toroid: $\mathrm{L}_{\mathrm{BT}-\mathrm{sh}}$ (it is assumed that $\mathrm{L}_{1} \geq \mathrm{L}_{2}$ ); $\mathrm{M}_{0}$ is the mutual inductance between these parts; $R_{\mathrm{S}}$ is the resistance of the short between conductors; $I_{1} I_{1}$ and $I_{2}$ are the currents in the corresponding sections of the windings; $t$ is real time. The circuit analysis is done for $\mathrm{R}_{\mathrm{s}}=10 \mathrm{~m} \Omega$ and $10 \mu \Omega$.

\section{B. Ramp Rate Limitations}

The overall barrel toroid inductance is $7.3 \mathrm{H}(5.1 \mathrm{H}+2.2 \mathrm{H})$ which imposes a maximum ramp rate of $3 \mathrm{~A} / \mathrm{s}$ at $20 \mathrm{~V}$. Let us 


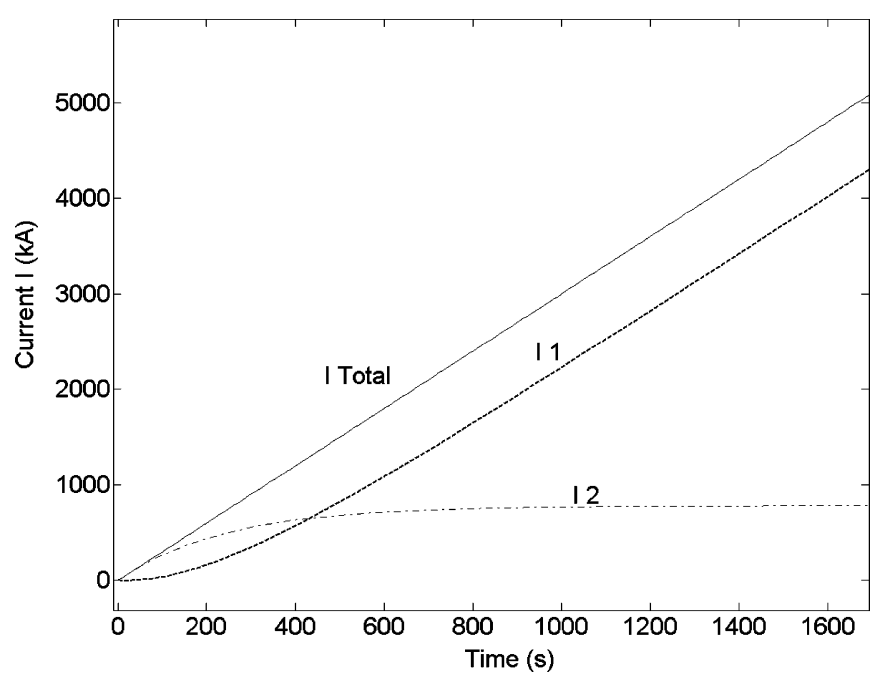

Fig. 2. Current versus time. Total current $\mathrm{I}_{\text {tot }}$, current $\mathrm{I}_{\text {Lshort }}=\mathrm{I} 1$ into short circuit coil. Current $\mathrm{I}_{\text {Rshort }}=\mathrm{I}_{2}$ into the short circuited resistance.

consider the charging of the Barrel Toroid with 4 shorted coils at rate

$$
\frac{d I}{d t}=3 \mathrm{~A} / \mathrm{s} \text {. }
$$

Typical time response of current into the short resistance and the inductance branches is shown in Fig. 2.

Kirchhoff's equations for this case can be written as follows:

$$
\left\{\begin{array}{l}
L_{2} \frac{\mathrm{d} I_{1}}{\mathrm{~d} T}+M o \frac{\mathrm{d} I}{\mathrm{~d} T}=I_{2} r_{s} \\
I_{1}+I_{2}=I(t)
\end{array}\right.
$$

The solution for the (1) was worked out by V. E. Keilin [3]. They are given by:

$$
\begin{aligned}
& i_{1}(t)=t-a \theta\left[1-\exp \left(-\frac{t}{\theta}\right)\right] \\
& i_{2}(t)=a \theta\left[1-\exp \left(-\frac{t}{\theta}\right)\right]
\end{aligned}
$$

where $i_{1}$ and $i_{2}$ are respectively the current in shorted BT coils and in the short-circuit resistance. Here

and

$$
a=1+k \sqrt{\frac{L_{1}}{L_{2}}}
$$

$$
k=\frac{M_{0}}{\sqrt{L_{1} L_{2}}}
$$

and the dimensionless parameter $\theta, \mathrm{i}_{1}, \mathrm{i}_{2}$ :

$$
\begin{aligned}
\theta & =\frac{L_{2} \dot{I}}{r_{s} I_{c}} \\
i_{1}(t) & =\frac{I_{1}}{I_{c}} \\
i_{2}(t) & =\frac{I_{2}}{I_{c}}
\end{aligned}
$$

where the critical current $I_{c}$ of the toroid conductors at $4.2 \mathrm{~K}$ and $5 \mathrm{~T}$ is $58 \mathrm{kA}$. Current in the shorted section can exceed the critical one and the winding will undergo a premature quench.

This corresponds to the case $\left|I_{2 \max }\right|>I_{c}$, thus $i_{2 m}>1$ i.e.,

$$
\theta>\theta^{*}=(a-1-\ln a)^{-1} \text {. }
$$

TABLE II

Circuit Data For 3 CASES OF SHORTS IN THE BT

\begin{tabular}{llcc}
\hline \hline $\begin{array}{l}\text { Cases of shorted } \\
\text { BT coils }\end{array}$ & $\begin{array}{l}\text { Short } \\
\text { resistance } \\
\text { value Rs }\end{array}$ & $\begin{array}{l}\text { Charging rate } \\
\text { ' } \theta \text { ' parameter }\end{array}$ & $\begin{array}{l}\text { Limit charging rate } \\
\text { parameter } \\
\theta^{*}=(a-1-\ln a)^{-1}\end{array}$ \\
\hline $\begin{array}{l}1-7 \text { configuration } \\
\mathrm{L}_{1}=6.85 \mathrm{H}\end{array}$ & $10 \mathrm{~m} \Omega$ & $2.8 \times 10^{-3}$ & 56.6 \\
$\mathrm{~L}_{2}=0.54 \mathrm{H}$ & $10 \mu \Omega$ & 2.8 & \\
$\mathrm{M}_{\mathrm{o}}=0.113 \mathrm{H}$ & & $2.3 \times 10^{-2}$ & \multirow{2}{*}{3253} \\
\hline $7-1$ configuration & $10 \mathrm{~m} \Omega$ & 23 & \\
$\mathrm{~L}_{1}=2.96 \mathrm{H}$ & & & \\
$\mathrm{L}_{2}=4.437 \mathrm{H}$ & $10 \mu \Omega$ & & \\
$\mathrm{M}_{\mathrm{o}}=0.113 \mathrm{H}$ & & $2.4 \times 10^{-2}$ \\
\hline 1 turn shorted & $10 \mathrm{~m} \Omega$ & $1.9 \times 10^{-7}$ & \\
$\mathrm{~L}_{1}=7.38 \mathrm{H}$ & & & \\
$\mathrm{L}_{2}=3.75 \times 10^{-5} \mathrm{H}$ & $10 \mu \Omega$ & $1.9 \times 10^{-4}$ & \\
$\mathrm{M}_{\mathrm{o}}=9.8 \times 10^{-4} \mathrm{H}$ & & & \\
\hline \hline
\end{tabular}

The calculations of the limit charging rate parameter are carried out for three cases of short circuits with potential short resistance values as displayed in Table II. It is shown that the current in the shorted coils will not exceed the critical one provided the ramp rate in the $\mathrm{BT}$ coils is less than $3 \mathrm{~A} / \mathrm{s}$.

\section{Thermal BeHAVIOR OF SHORT-CIRCUIT}

An FEA ANSYS model is developed to determine the maximum acceptable steady heat generation in the conductors in presence of short-circuited turns.

\section{A. Thermal Consequences of a Short}

Ramping up a magnet exhibiting short-circuited turns can cause a premature quench due to the local heating. The time constant of the heat diffusion must then be compared with the ramping time to see the consequences.

The characteristic thermal time constant of quench propagation is defined as

$$
\tau_{h}=\left(\frac{C \gamma}{k}\right) M P Z^{2} .
$$

Taking the Minimum zone Propagation length MPZ equal to $0.5 \mathrm{~m}$ at $10 \mathrm{kA}$ for ATLAS conductors the longitudinal characteristic diffusion time constant $\tau_{\mathrm{h}}$ is of the order of 1 second. The stationery heat state is then reached locally in the conductors in about 4 seconds which is much less than the overall charging time of about 6200 seconds. Therefore, it is necessary to investigate the maximum spot temperature generated at the short region by solving the stationary nonlinear heat propagation differential equations assuming a certain steady power generated by the induced current in the short.

The temperature profile of the hot spot at the short-circuit location is governed by the heat equation at time $\mathrm{t}$ :

$$
\begin{aligned}
\frac{\partial}{\partial x}\left(k A\left(\frac{\partial T}{\partial x}\right)\right)+ & \frac{\partial}{\partial y}\left(k A\left(\frac{\partial T}{\partial y}\right)\right)+\frac{\partial}{\partial z}\left(k A\left(\frac{\partial T}{\partial z}\right)\right)- \\
& \gamma C A \frac{\partial T}{\partial t}-h P\left(T-T_{0}\right)+Q_{h}=0
\end{aligned}
$$

where $\gamma C$ is density $\gamma$ times specific heat $\mathrm{C}_{\mathrm{p}}$, $\mathrm{A}$ is the conductor cross sectional area, $\mathrm{h}$ is the insulation-conductor heat transfer coefficient, $\mathrm{P}$ the perimeter of the conductor in contact 
TABLE III

MATERIAL PROPERTIES

\begin{tabular}{|c|c|}
\hline Characteristics & Value \\
\hline$\overline{\mathrm{K}_{\text {Alu }} @ 4.5 \mathrm{~K}(\mathrm{RRR}=1000)}$ & $2000 \mathrm{Wm}^{-1} \mathrm{~K}^{-1}$ \\
\hline Cp Alu@4.5K & $844 \mathrm{~J} / \mathrm{m}^{3} \cdot \mathrm{K}$ \\
\hline $\mathrm{Dth}_{\mathrm{Alu}}=\mathrm{K} / \mathrm{Cp}$ & $2.5 \mathrm{~m}^{2} \mathrm{~s}^{-1}$ \\
\hline $\mathrm{K}_{\mathrm{G} 10} @ 4.5 \mathrm{~K}$ & $65 \mathrm{mWm}^{-1} \mathrm{~K}^{-1}$ \\
\hline $\mathrm{Cp}_{\mathrm{G} 10} @ 4.5 \mathrm{~K}$ & $4800 \mathrm{~J} / \mathrm{m}^{3} \mathrm{~K}$ \\
\hline $\operatorname{Dth}_{\mathrm{G} 10}=\mathrm{K} / \mathrm{Cp}$ & $1.35 \times 10^{-5} \mathrm{~m}^{2} \mathrm{~s}^{-1}$ \\
\hline
\end{tabular}

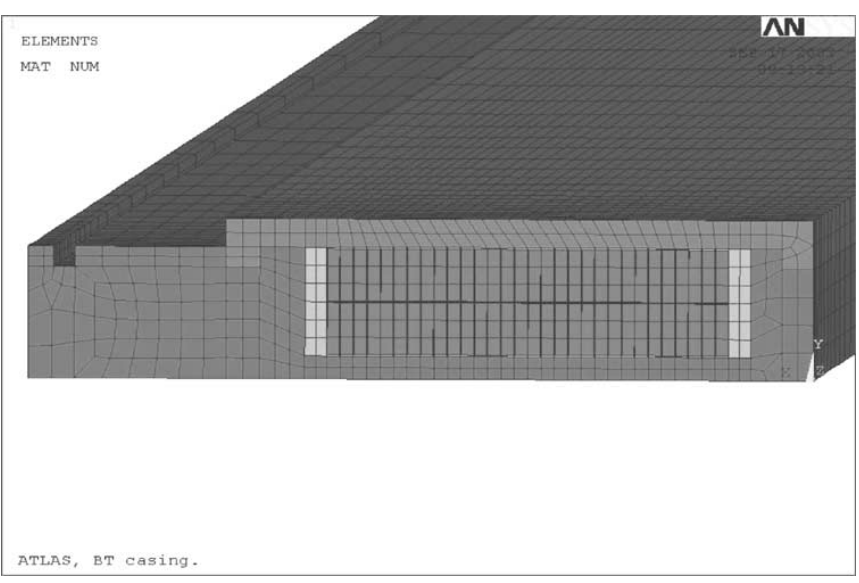

Fig. 3. ANSYS BT coil cold mass equivalent model.

with cooling surface, $\mathrm{Q}_{\mathrm{h}}$ is the Joule heating power inside the short-circuit. The time-independent state equation is given by removing the dT/dt term. Table II summarizes the main material properties considered in the barrel conductors (Table III).

A 3D FEA ANSYS thermal model is developed with real geometry of BT cold mass (see cross section shown in Fig. 3) to evaluate the maximum local stationery temperature. The steady state heat deposited at the short-circuit at instant $t$ is evaluated as:

$$
Q_{h}=\frac{\mathrm{U}_{\mathrm{s}}^{2}}{R_{s}}=\frac{\left(L \cdot \frac{d I_{2}}{d t}+M \frac{d I}{d t}\right)^{2}}{R_{s}}=\frac{\left(M \frac{d I}{d t}\right)^{2}}{R_{s}}
$$

where $\mathrm{I}$ is the main supply current and $\mathrm{I}_{2}$ the current into the short circuit.

Fig. 4 shows the calculated temperature rise at the inner turns short circuit as function of heat power source.

The maximum stationary temperature due to heating in a short between inner turns of the double pancake windings is compared with the current sharing temperature of the ATLAS BT conductors of $6.5 \mathrm{~K}$ at $3.8 \mathrm{~T}$ representing the quench initiation. From the thermal analysis, it appears that the maximum corresponding heating power during ramping up is $\mathrm{Q}_{\mathrm{h} \max }=24 \mathrm{~W}$, which corresponds to a minimum acceptable short resistance value of $5 \mathrm{~m} \Omega$ in the case of a single short circuited coil and $0.3 \mu \Omega$ for a single shorted turn. When the number of shorted turns or coils increases, the limit value of short resistance increases and the operating ramping rate has to be reduced according to (9). It is demonstrated that in most cases, shorted coils will lead to a quench due to local heating of the conductors when the current is ramped up.

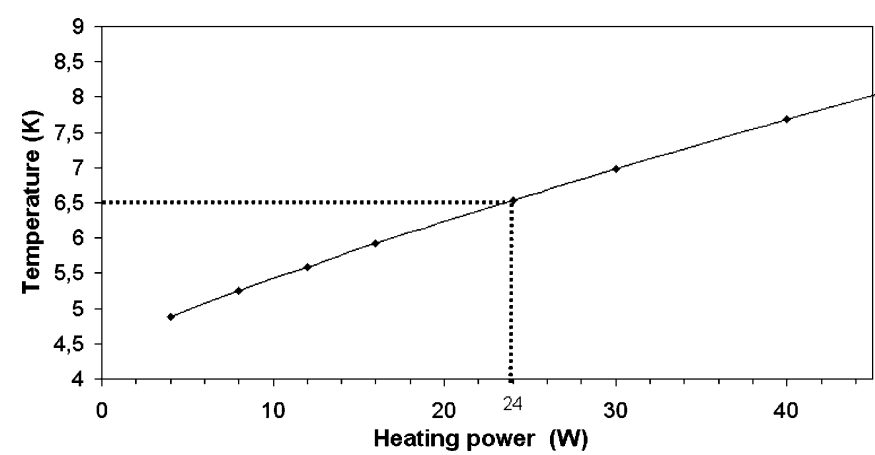

Fig. 4. Maximum steady temperature generated by short circuit between inner turns-power corresponding to sharing temperature.

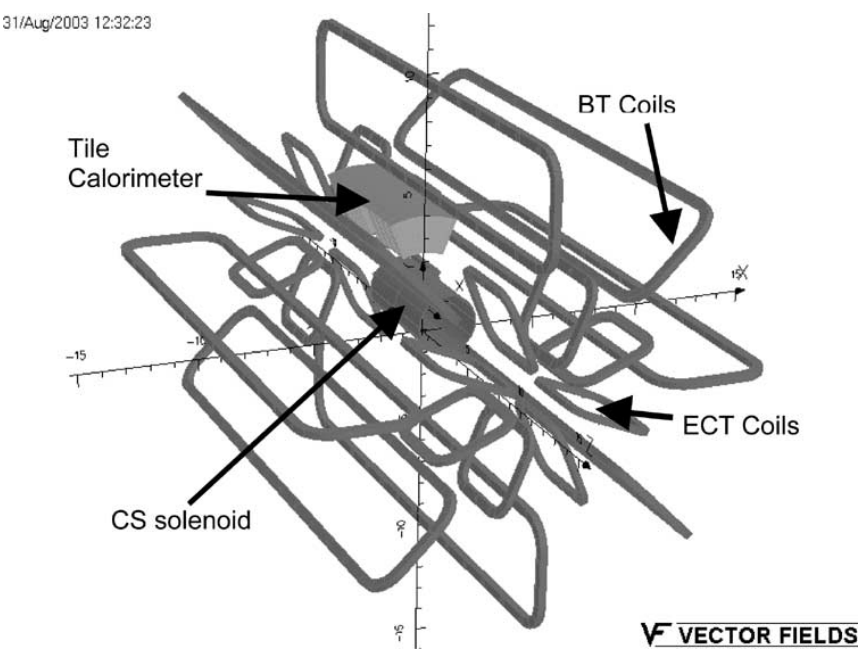

Fig. 5. Electro-magnetic model in Opera of the toroids.

\section{Mechanical Models}

\section{A. 3D Mechanical Model}

A global model is developed using Opera to derive the force distribution along conductors in various failure scenarios. The calculations are performed both in presence and absence of the iron yoke in the tile calorimeter but always without the solenoid. This allowed using a global symmetry about $\mathrm{z}=0$ plane and one in the plane of symmetry of the shorted coil with respective tangential and normal field boundary conditions.

The model as illustrated in Fig. 5. has about 150000 nodes and is optimized by refining the mesh around the conductors region. This improves accuracy of the integration of Lorentz forces. It is checked that $90 \%$ of the EM forces on the conductors are generated by BT and ECT coils in air whereas the contribution of solenoid is estimated less than $10 \%$. To represent the real stack of laminated iron plates by a homogeneous material an adaptation factor in the formulae [4] has been used: The $B-H$ curve was multiplied by a factor of 0.658 while the packing factor was set as 0.855 .

\section{Mechanical Behavior of Short-Circuited BT CoILS}

The coils in the barrel toroid in normal operation are subject to balanced radial forces. The occurrence of a short circuit in one or more coils breaks the symmetry and some unbalanced tangential forces appear. The distribution of radial and 


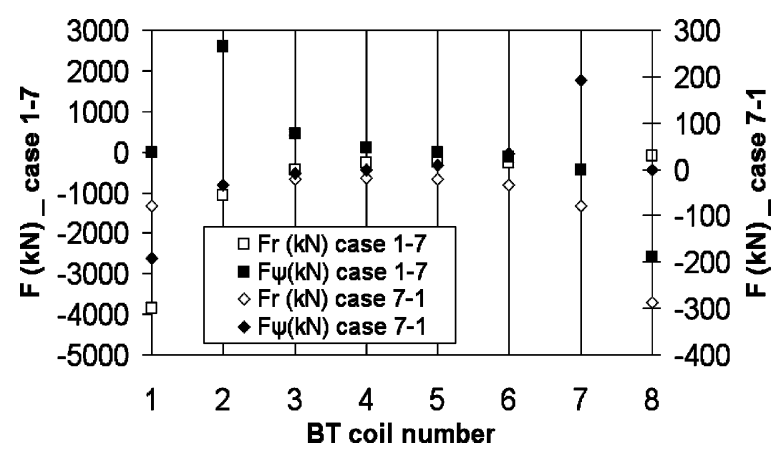

Fig. 6. Maximum resultant forces on BT coils in 1-7 and 7-1 short-circuit configurations.

azimuthal forces along the BT and ECT conductors is investigated in the case of 1-7, 2-6 and 4-4 shorted BT coils configurations and compared with normal operation. The analysis is important to understand changes in the radial forces distribution through tie rods and other coil supports. Previous quench circuit analysis studies with shorted coils [5] have addressed the consequences of failures of quench heaters. Our analysis is meant to obtain instantaneous force distributions at times when the tangential force in the BT conductors is maximum corresponding to a given peak current difference $\Delta$ IBTsh - BTnsh.

The cases discharge of heater failure has shown disruptive resultant azimuthal forces within the BT in the 4-4 short circuit coils configuration. However this will not be further analyzed since full quench heater failure has a negligible probability as there is redundancy on quench firing safety device. All resultant force results in the case of short-circuit configurations are verified by using Biot and Savart based on infinite straight conductors model depicted by Acerbi [6]. Fig. 6 illustrates the radial and tangential resultant calculated forces in the cases 1-7 and $7-1$.

This allowed validating results only for the cases that did not model the influence of the tile calorimeter. Discrepancies in radial forces with the Opera model are due to the fact that the mentioned method did not include the influence of the ECT while estimating the force on the BT coils, which was found to be significant in some cases. A maximum error of $10 \%$ was noticed between Biot and Savart calculations and the FEA model results.

\section{RESULTS}

\section{A. Force Distribution in Nominal Operation}

Fig. 7 shows the distribution along the BT conductors of the radial forces $F_{r}$ and longitudinal $F_{z}$ per unit length in normal operating conditions at $20.5 \mathrm{kA}$ in presence of Tile calorimeter, Central solenoid and ECT. The peak value of the total force defined as

$$
F_{t}=\sqrt{F_{r}^{2}+F_{z}^{2}}
$$

is reached at $11.4 \mathrm{~m}$ from the center of straight conductors $F_{t}=2.5 \mathrm{MN} / \mathrm{m}$. Due to the presence of the iron in the Tile Calorimeter and the solenoid stray field the peak force reduced by $7 \%$.

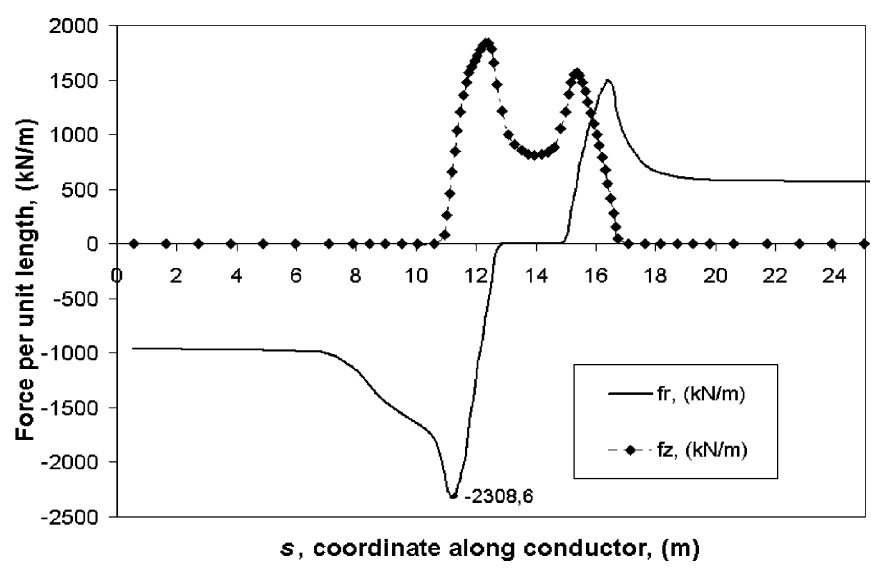

Fig. 7. Specific radial and longitudinal forces on BT coils in normal operating condition with Tile, CS, ECT.

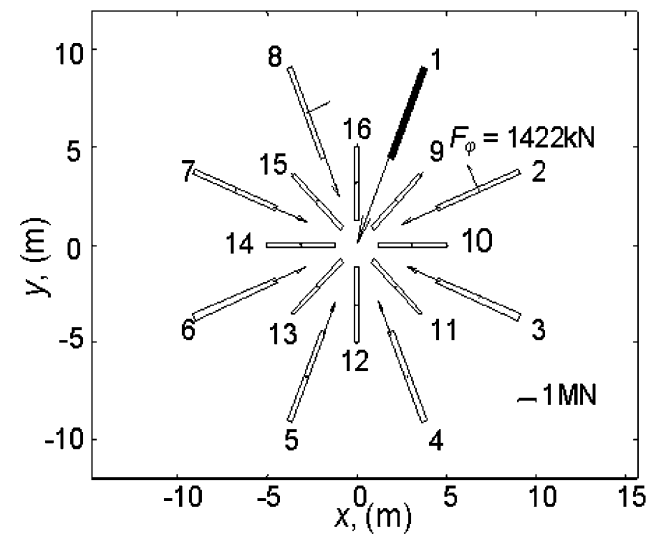

Fig. 8. Resultant forces on BT and ECT coils with one BT coil short-circuited.

The resultant radial force acting on each coil is $F_{r}=12.7 \mathrm{MN}$. The fringing field of the solenoid induced a very light tangential loading, which reaches a maximum magnitude for $f_{\varphi}=-19 \mathrm{kN} / \mathrm{m}$ at $s=5.977 \mathrm{~m}$.

\section{B. One BT Coil Short-Circuited}

In the calculation without the iron yoke, the maximum radial force is found at the shorted coil with a value $F_{r}=-4.7 \mathrm{MN}$ and the maximum tangential force is acting on the neighboring coil, with value $F_{\varphi}=1.4 \mathrm{MN}$, as shown in Fig. 8 .

Including the calorimeter iron yoke in the calculation increases the magnitude of $F_{r}$ by $18 \%$, and $F_{\varphi}$ by $24 \%$ on the most tangentially loaded BT racetrack.

Force differences appear to be concentrated on the inner straight region of the conductor. The force distribution over the most tangentially loaded BT racetrack (Fig. 9) shows that the tangential force curve is quite smooth, with values between 18 and $70 \mathrm{kN} / \mathrm{m}$.

\section{Four BT Coils Short-Circuited}

The maximum radial force $F_{r}=-17.8 \mathrm{MN}$ is found on the two racetracks that were farther away from the shorted ones. Maximum tangential forces acted on the two nonshorted racetracks that are closest to the shorted ones $F_{\varphi}=-6.2 \mathrm{MN}$. The force distribution shows a smooth tangential force curve 


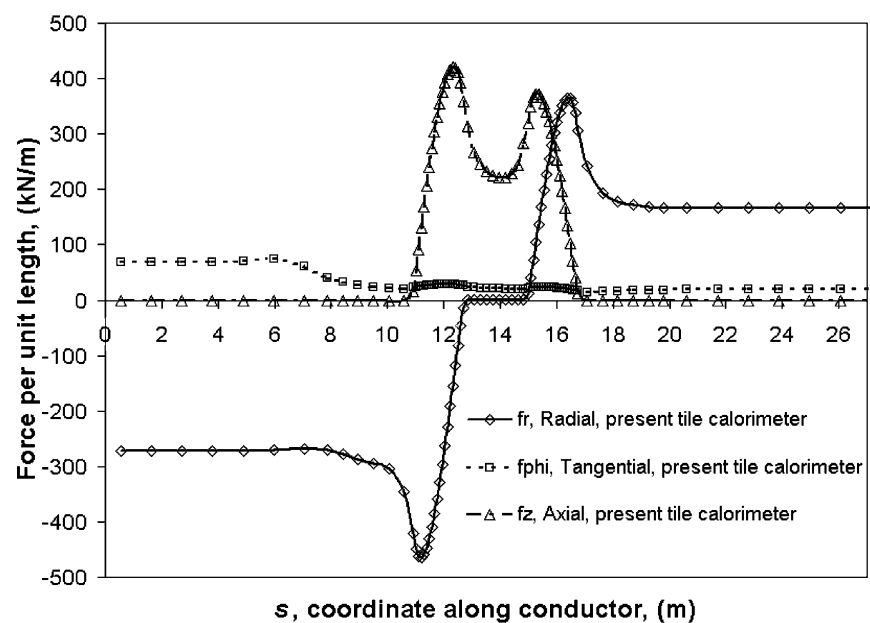

Fig. 9. Specific forces along BT conductors on the most tangentially loaded BT coil with one short-circuited coil (1-7 configuration).

$\mathrm{F} \varphi$, reaching a maximum magnitude of $250 \mathrm{kN} / \mathrm{m}$ on the inner straight conductor at coordinate $s=5.9 \mathrm{~m}$.

\section{Seven BT Coils Short-Circuited}

The maximum radial force acts on the nonshorted coil, with $F_{r}=-3.5 \mathrm{MN}$. The maximum tangential force $F_{\varphi}$ is found on the neighboring conductors equals $190 \mathrm{kN}$, which is confirmed by analytical calculations within $4 \%$.

\section{E. BT-ECT Interaction Forces}

The longitudinal interaction force between BT and ECT's with a magnitude of $2.8 \mathrm{MN}$ in the nominal case, is decreased to $160 \mathrm{kN}$ in the case of one shorted BT coil. The resultant radial component on ECT is directed toward the plane of symmetry of the BT shorted coils. In Table IV the maximum resultant radial and azimuthal force are reported.
TABLE IV

\begin{tabular}{|c|c|c|c|}
\hline \multirow{2}{*}{$\begin{array}{l}\text { Short } \\
\text { Configurations }\end{array}$} & \multirow{2}{*}{$\begin{array}{l}\text { Fr resultant } \\
\text { on BT } \\
(\mathrm{MN}) \\
\end{array}$} & \multicolumn{2}{|c|}{ Maximum F $\varphi$ on FBT-ECT } \\
\hline & & BT $(\mathrm{MN})$ & $\begin{array}{l}\text { Resultant } \\
\text { (MN) }\end{array}$ \\
\hline No short & 0 & 0 & -2.8 \\
\hline $1-7$ & -4.6 & 1.4 & -0.16 \\
\hline $4-4$ & -17.8 & 6.2 & -0.74 \\
\hline $7-1$ & -3.5 & 0.19 & -0.4 \\
\hline
\end{tabular}

\section{CONCLUSION}

Limitations for the ramp rate in the ATLAS magnet system have been defined in presence of short circuited coils. The electromagnetic force distributions in the Toroids are investigated for several possible fault scenarios.

- Radial and tangential force distributions were obtained for the worst instantaneous condition in each of three failure scenarios. The results agree with earlier data and safe operation of the cold mass suspension system is confirmed.

The effect of neighboring iron in the Tile Calorimeter was examined as well and checked as a safe configuration.

- For the short circuit cases investigated, adding the tile calorimeter in the simulation are found to increase the overall tangential force on the most tangentially loaded conductor by about $24 \%$. This corresponded to a force distribution change located on the inner axial straight of the conductor.

\section{REFERENCES}

[1] ATLAS Barrel Toroid TDR, CERN/LHCC/97-19, 1997.

[2] OPERA-3D User Guide, Vector Fields Ltd., Kidlington, Oxford.

[3] V.E. Keilin, "Analysis of the behavior of superconducting windings with short circuited turns," IEEE Trans. Appl. Supercond., vol. 11, no. 1, pp. 1454-1456, March 2001.

[4] F. Bergsma, Calculation of the Average Magnetic Permeability of the ATLAS Tile Calorimeter, CERN/PPE-EC, April 1997.

[5] G. Volpini, Quench in ATLAS Under Faulty Conditions, INFN-Sezione di Milano, no. Internal report LASA/ATLAS/160, June 8, 2003.

[6] E. Acerbi and M. Sorbi, Calculation of the Forces in the Coils of the ATLAS Barrel Toroid and Their Simulation in the B0 Model With a Magnetic Mirror, Dipartimento di Fisica dell'Universita' degli Studi di Milano, INFN-Sezione di Milano, October 2, 1996. 